\title{
Effective preparation of graphite nanoparticles using mechanochemical solid-state reactions ${ }^{1}$
}

\author{
S. Motozuka $^{a, *}$, M. Tagaya ${ }^{b, * *}$, N. Ogawa ${ }^{c}$, K. Fukui $^{a}$, \\ M. Nishikawa ${ }^{b}$, K. Shiba ${ }^{d}$, T. Uehara ${ }^{a}$, and T. Kobayashi ${ }^{b}$ \\ ${ }^{a}$ Department of Mechanical Engineering, Gifu National College of Technology, \\ 2236-2 Kamimakuwa, Motosu, Gifu 501-0495, Japan \\ ${ }^{b}$ Department of Materials Science and Technology, Nagaoka University of Technology, \\ 1603-1 Kamitomioka, Nagaoka, Niigata 940-2188, Japan \\ ${ }^{c}$ Atmosphere and Ocean Research Institute, University of Tokyo, \\ 5-1-5 Kashiwanoha, Kashiwa, Chiba 277-8564, Japan \\ ${ }^{d}$ World Premier International Research Center,
} International Center for Materials Nanoarchitectonics, National Institute for Materials Science, 1-1 Namiki, Tsukuba, Ibaraki 305-0044, Japan

* Corresponding author:

Satoshi Motozuka, Ph.D.

e-mail: motozuka@gifu-nct.ac.jp

tel: +81-58-320-1379

affiniation: Department of Mechanical Engineering,

postal address: Gifu National College of Technology, Gifu 501-0495, Japan

** Corresponding author:

Motohiro Tagaya, Ph.D.

e-mail: tagaya@mst.nagaokaut.ac.jp

tel: +81-258-47-9345

affiniation: Department of Materials Science and Technology,

postal address: 1603-1 Kamitomioka, Nagaoka, Niigata 940-2188, Japan. 
2 The mechanochemical milling was utilized to control the nanostructures of plate-like graphite

3 (Gp) microparticles in an argon atmosphere and at room temperature. The aggregated Gp

4 nanoparticles were prepared by the simple solid-state process without organic solvent. Electron

5 microscope observations, X-ray diffraction patterns and Raman spectra revealed the fractured

6 plate-like Gp microparticles as well as the Gp microparticles agglomerated to each other through

7 the dissociative edge surfaces by the milling process to finally generate the Gp nanoparticle

8 aggregates. In the process, the ratio of the edge plane surfaces with the dangling bondings to

9 layered internal surfaces clearly increased with the milling. Therefore, the low environmental

10 burden process for preparing the Gp nanoparticle aggregation was achieved.

\section{Keywords}




\section{1. Introduction}

2 Graphite (Gp) is widely used as an anode active material of lithium-ion batteries in most

3 commercial products, and the nanoparticle aggregation (nanoporous solid) state is focused

4 because of the conductivity as well as the specific surface area. Recently, the Gp nanoparticle-

5 aggregated electrodes are functionalized by composting with other elements such as iron and

6 copper in order to achieve high performance. The crystallographic structures, surface

7 morphologies, and physicochemical parameters of Gp have a significant influence on the

8 properties of such composites doped with other elements. Thus, the composite techniques for Gp

9 have been widely investigated [1,2]. The techniques involving an organic binder are mainly used

10 for enhancing the interfacial interactions [3, 4]; however, it decreases mechanical properties and

11 contaminates a furnace during the sintering process. Therefore, the preparation of nanoparticle

12 aggregation as well as the development of a new Gp surface modification without an organic

13 binder is desired.

14 Gp has plate-like morphology and layered structure with crystalline carbon networks [5], 15 which are stabilized by the end $\mathrm{C}-\mathrm{H}$ groups [6]. The terminal hydrogen atoms can be removed 16 by thermal heating at $700-900{ }^{\circ} \mathrm{C}$. The generated dangling bondings subsequently form the

17 bondings among the end surfaces, resulting in nanographites with single/double-walled carbon

18 nanotubes and graphene [7]. Thus, the dissociation of the $\mathrm{Gp}$ end groups will generate the

19 activated Gp surfaces with dangling bondings [8], which would form the unique morphologies.

20 A new approach to control the $\mathrm{Gp}$ morphologies and nanostructures is termed a

21 mechanochemical milling technique in this study. The force-induced defects and dangling

22 bondings in the lattice will provide the composites doped with other materials. We have already 
1 proposed that the control of surface structures with a mechanochemical methodology, which is a

2 powerful tool to modify a solid state material and to obtain new defect states unachievable by

3 other means, is an important issue. Such control is mainly stimulated by modern materials

4 science, the needs of industry for new nanomaterials and green technologies [9-13]. The goal of

5 the mechanochamical reactions is determining the major factors influencing the dynamics of the

6 process for compositing heterogenetic atoms $[10,11]$, as well as revealing the possibilities to

7 regulate the structure and properties of a product, that is, the traditional goal of solid state

8 chemistry to adjust the reactions in time and space [13]. Therefore, these controllable defects and

9 the formation of dangling bondings in the lattice structure will provide novel activation

10 properties as well as nanomorphologies.

11 In this study, the mechanochemical milling control of the nanostructures of plate-like Gp

12 microparticles in an argon atmosphere and at room temperature was conducted for the first time.

13 The surface chemical states of the activated Gp were investigated to clarify the fracture and

14 aggregation mechanism among the mechanochemicaly-activated Gp nanoparticle surfaces.

\section{2. Experimental}

\section{2.1. Preparation of Gp Nanoparticles}

18 The experimental set-up for the mechanochemical solid-state reaction and photographs during

19 the milling are shown in the Supporting Information, Scheme S1. The milling vessel made of a

20 stainless steel rod (SUS304) was used and was surrounded by a water jacket in order to suppress

21 the heat generated and keep room temperature $\left(20-25^{\circ} \mathrm{C}\right)$ during the milling. Twenty SUJ2 steel 
1 balls (AISI52100) with the diameter of $10 \mathrm{~mm}$ were used as the milling medium. The detailed

2 experimental set-up was described in our previous reports [10,11].

3 First, a $0.2 \mathrm{~g}$ sample of plate-like Gp microparticles (average diameter: ca. $10 \mu \mathrm{m}$, purity: $>99$

$4 \%$, Nihonkokuenkogyo Co., Ltd.), which was determined by the optimum Gp volume to medium

5 balls for efficient milling, was added into the milling vessel. The vessel was mounted on a

6 centrifugal ball mill (NEV-MA-8, Nisshingiken Co., Ltd.) and stored under vacuum for 1 day in

7 order to remove water molecules and other substances adsorbed on the surfaces. Then, dry argon

8 gas (purity: $>99.999 \%$ ) was introduced into the milling vessel until the pressure increased to

$9203 \mathrm{kPa}$. This process was repeated three times by completely replacing the argon. The vessel

10 was then immediately swung at the speed of $59.1 \mathrm{rad} / \mathrm{s}$ for $8 \mathrm{~h}$ under room temperature.

11 The kinetic energy transfer from the media to the Gp particles was calculated by the following

12 equations. The medium moves in a circular path against the inside wall of the vessel to

13 subsequently press against the wall by centrifugal force. As a result, the kinetic energy transfer

14 from the media to the particle occurs when the balls compress the trapped particles between the

15 balls and the wall. The compressed force $(F)$ can be expressed by equation (1),

$$
F=m r \omega^{2}
$$

17 where $m$ is the mass of the ball, $r$ is the orbit radius of the ball movement and $\omega$ is the milling 18 rate. $F$ can be regarded as the working force on the particle surfaces, and was calculated using $m$ 19 of $3.5 \times 10^{-3} \mathrm{~kg}, r$ of $2.7 \times 10^{-2} \mathrm{~m}$ and $\omega$ of $40 \mathrm{rad} / \mathrm{s}$ to be $1.5 \times 10^{-1} \mathrm{~N}$. Therefore, the kinetic 20 energy transfer to the media can be expressed by equation (2), 
2 where $E$ is the kinetic energy, $n$ is the number of balls, $v$ is the circumferential velocity that can

3 be obtained for the product of the orbit radius $r$ and the milling rate $\omega$. $E$ was calculated using $n$

4 of 20 and $v$ of $1.1 \mathrm{~m} / \mathrm{s}$ to be $4.1 \times 10^{-2} \mathrm{~J}$.

\section{$5 \quad 2.2$ Characterization}

6 The resultant Gp particles were characterized by field emission scanning electron microscopy

7 (FE-SEM), transmission electron microscopy (TEM), X-ray diffraction (XRD), and Raman

8 spectroscopy.

9 The morphology of the Gp particles was observed using a FE-SEM (S4800, Hitachi Co., Ltd.)

10 at an accelerating voltage of $15 \mathrm{kV}$. The detailed nanostructures based on bright-field and dark-

11 field TEM and selected area electron diffraction (SAED) images were obtained and analyzed

12 using a JEM-1400 (JEOL Co., Ltd.) at an accelerating voltage of $120 \mathrm{kV}$. DF-TEM images from

13 Gp (002) diffraction were observed using an objective aperture. Selected DF-TEM images (from

14 images covering $0-180^{\circ}$ of the diffraction ring) were superimposed using software

15 (DigitalMicrograph ${ }^{\circledR}$; Gatan, Inc., Pleasanton, CA, USA) (for details, see Supporting

16 Information, Fig. S2). The preparation of the samples before observation was done by dispersing

17 a small amount of the Gp particles into ethanol, casting the suspension onto a carbon-coated 18 copper grid, and then drying.

19 The XRD patterns were recorded by a Smartlab (Rigaku Co., Ltd.) using monochromatized $20 \mathrm{CuK} \alpha$ radiation. The primary optics was equipped with a solar slit $\left(5.0^{\circ}\right)$, a divergence slit $\left(0.5^{\circ}\right)$ 
1 and a scattering slit $\left(0.5^{\circ}\right)$. The secondary optics was equipped with a first receiving slit $(0.08$

$2 \mathrm{~mm})$, a Gp monochrometer, a second receiving slit $(0.13 \mathrm{~mm})$ and a detector.

3 The Raman spectra were recorded by a NRS-7200 (JASCO Co., Ltd.) equipped with a

4 holographic grating (L400) and $\mathrm{Nd}: \mathrm{YVO}_{4}$ laser at the wavelength of $532 \mathrm{~nm}$ for the excitation.

5 The laser power was $3.56 \mathrm{~mW}$ to avoid any sample degradation. The exposure time, resolution,

6 scan times and measured wavenumber were $4 \mathrm{~s}, 6.8 \mathrm{~cm}^{-1}, 100$ times and $100-1800 \mathrm{~cm}^{-1}$,

7 respectively. Based on the Gaussian function, the spectra were fitted to each of the characteristic

8 bands $\left(D, G\right.$, and $\left.D^{\prime}\right)$. In the deconvolution, the components, number of peaks, and peak

9 positions and widths were initially fixed according to previous reports [14, 15], and then were

10 refined only for the peak heights. During the final optimization, only the components and peak

11 widths were refined again to reduce the residual values up to less than $1.0 \times 10^{-4}$ in order to

12 obtain the separated spectra. In the resulting spectra, the raw spectra, the deconvoluted spectra

13 and the summation were represented by black, green and red spectra color curves, respectively,

14 as shown in Fig. 3 (a). In the deconvoluted spectra, the integrated intensity ratios of the $D$ band

15 to $G$ band $\left(I_{D} / I_{G}\right)$ and of the $D^{\prime}$ band to $G$ band $\left(I_{D}{ }^{\prime} / I_{G}\right)$ were calculated, leading to the

16 investigation of the lattice disorder as well as the formation of nanocrystalline $\mathrm{Gp}$.

17 X-ray photoelectron spectroscopy (XPS) spectra of the particle surfaces were measured by a

18 PHI5000 Versa Probe (ULVAC-PHI, Inc.) using a monochromatized Al X-ray source (Al

$19 \mathrm{Ka}$ line at $1.4866 \mathrm{keV}$ ) at $15 \mathrm{kV}$ and $25 \mathrm{~mW}$. The $\mathrm{C} 1$ s spectra were measured in the ranges

20 between $282-287 \mathrm{eV}$ and the detailed characterization procedure is described in the Electronic

21 Supplementary Materials, XPS Characterization Procedure. 


\section{Results and discussion}

2 Fig. 1 shows the representative FE-SEM and TEM images of the Gp microparticles without 3 milling and milled for 1 and $8 \mathrm{~h}$. The Gp microparticles without milling exhibit a plate-like shape

4 with the particle size of $2-10 \mu \mathrm{m}$ which circumferentially consists of flake-shaped crystals (Fig.

$51(\mathrm{a}-\mathrm{c}))$. Here, the Gp crystalline structure is one in which the carbon atoms lie in the six-

6 membered ring honeycomb network structure sheets with extremely strong covalent bonds

7 between the atoms inner each sheet. One layer of the sheet is well-known as graphene. The plane

8 corresponds to (001) in the Miller index called the basal plane [16]. The end of the six-membered

9 ring structure is called the edge plane, exhibiting the active surfaces such as $-\mathrm{COOH},=\mathrm{C}=\mathrm{O}$, and

10 so on. Therefore, the larger plate-like morphologies at the initial milling stage indicates that the

11 inactive layered graphene sheets were dominant as compared with the active edge surfaces.

12 During the mechanochemical milling for $1 \mathrm{~h}$, cracks were newly formed parallel to the plate

13 plane (i.e., c-plane) as shown in Fig. 1 ( $\mathrm{d}$ and e). The partially-fragmented plates were

14 aggregated and stacked as shown in Fig. 1 (f). In appearance, the plate-like shaped particles are

15 preserved after the milling for $1 \mathrm{~h}$. However, as shown in Supporting Information Fig. S2 (BF-

16 TEM and DF-TEM images with SAED), the arbitrary SAED spots in the Debye rings based on

17 the (002) diffraction patterns indicate the polycrystalline structures with different orientations.

18 The size of crystallites is on a nanometer scale (ca. $100 \mathrm{~nm})$, which corresponds to the domain

19 sizes formed by the cracking in the TEM image (Fig. 1 (f)).

20 During the milling for $8 \mathrm{~h}$, the plate-like shapes completely changed to nanoparticulate

21 structures with the average size of ca. $50 \mathrm{~nm}$, and the nanoparticles were aggregated as shown in

22 Fig. $1(\mathrm{~g}-\mathrm{i})$. Gp nanoparticles were formed by the mechanochemical milling process, while 
1 preserving the aggregation state. Generally, it is known that the milling in an inert atmosphere

2 induces an effective milling which can form smaller size particles as compared to those form in

3 reactive atmospheres (e.g., $\mathrm{O}_{2}, \mathrm{H}_{2}$ ) [16]. In fact, we also conducted the milling in dried $\mathrm{O}_{2}$ and

4 air. As a result, the plate-like particle morphology and size did not change, indicating no

5 aggregation and fragmentation among the particles in the atmospheres because of the smaller

6 friction coefficient by the adsorbed oxygen gas at the interfaces. This suggests the importance of

7 the milling in an inert atmosphere. The inert atmosphere enhances the aggregation among the

8 newly-generated active surfaces with the dangling bondings by the milling, enabling an effective

9 milling $[10,11,17]$. On the other hand, the active atmosphere reacts with the newly-generated

10 surfaces to immediately reach an equilibrium milling state without aggregation [17]. That is

11 because the dispersion state significantly provides a lubrication effect due to the slipping of basal

12 plane during the milling, forming the larger size particles [18]. Therefore, it was suggested that

13 the present simple milling process in nitrogen atmosphere induces the aggregation state among

14 the newly-generated Gp edge surfaces with dangling bondings.

15 After the milling, we took out the samples from the chamber to air and then measured XPS

16 spectra. The C 1s XPS spectra after the milling were divided into two peaks attributed to the

$17 \mathrm{C}-\mathrm{C}(284.8 \mathrm{eV})$ as well as $\mathrm{C}-\mathrm{O}(286.1 \mathrm{eV})$. The peak positions and intensities are almost the

18 same irrespective of the time (data not shown). In this case, the $\mathrm{C}-\mathrm{C}$ bonding is attributed to the

19 Gp framework structures. Given that a reaction occurred in air, the $\mathrm{C}-\mathrm{O}$ bonding might be

20 similar to the oxidized layer of Gp, indicating the formation of dangling bonds on the end of Gp.

21 An NMR study has revealed that Gp oxides contain aromatic regions randomly interspersed with

22 oxidized aliphatic six-membered rings, and the oxidized rings contain $\mathrm{C}-\mathrm{O}-\mathrm{C}$ (epoxide) and

$23 \mathrm{C}-\mathrm{OH}$ groups, while the sheets terminate with $\mathrm{C}-\mathrm{OH}$ and $-\mathrm{COOH}$ groups [30]. In this study, the 
1 oxidation groups formed in air imply the formation of dangling bondings at the Gp edge

2 surfaces.

3 Fig. 2 shows the XRD patterns of the Gp microparticles without milling and milled for $0.5 \mathrm{~h}, 1$

$4 \mathrm{~h}, 2 \mathrm{~h}$ and $8 \mathrm{~h}$. All the diffraction patterns exhibit the Gp hexagonal and rhombohedral phases.

5 Here, the interlayer bondings among the graphene sheets mutually work with the weak

6 interactions. The $A B A B \ldots$ stacking sequence results in the hexagonal crystal symmetry, $D 6 \mathrm{~h}$

7 [19]. Furthermore, an alternative $A B C A B C \ldots$ stacking sequence based on the rhombohedral

8 symmetry is known to be in the present defective Gp, always as a mixture with the hexagonal

9 phase [20]. In Fig. 2 (a), peaks at $26.6^{\circ}$ and $54.7^{\circ}$ attributed to the hexagonal interlayer spacing

10 (001) ((002) and (004)) were clearly observed [20]. The peak shapes were broaden with

11 increasing reaction time. In Fig. 2 (b), peaks at $42.4^{\circ}, 43.4^{\circ}, 44.6^{\circ}, 46.2^{\circ}, 50.7^{\circ}, 59.9^{\circ}, 72.6^{\circ}$,

$1277.5^{\circ}, 82.6^{\circ}$ and $87.1^{\circ}$ due to the hexagonal (100), rhombohedral (101), hexagonal (101),

13 rhombohedral (012), hexagonal (102), hexagonal (103), hexagonal (104), hexagonal (112),

14 hexagonal (105) and hexagonal (106), respectively, were also observed [20].

15 In the peaks between $2 \theta=42-47^{\circ}$, the studies on non-graphitic carbons have determined the

16 (100) reflections of randomly stacked graphene sheets [25], suggesting that the milled Gp

17 particles in the present study partially consist of randomly stacked graphene sheets at the final

18 milling stage. During the milling, the $2 D$ arrangement in the graphene planes was preserved,

19 whereas the $3 D$ arrangement was gradually destroyed. It can be explained by the different

20 interactions based on the strong $\mathrm{sp}^{2} \mathrm{C}-\mathrm{C}$ covalent bondings. These bondings make up the

21 graphene planes with the weak van der Waals forces that stack these planes together $[16,25]$. In

22 other words, the preferential cleavages along the weak interlayer bondings disrupt the layered 
1 structures as well as the $\mathrm{C}-\mathrm{C}$ networks in the plane by the milling. Based on these viewpoints,

2 the (002) diffraction peak position slightly changes from $26.6^{\circ}$ to $26.5^{\circ}$ after the milling for $8 \mathrm{~h}$,

3 indicating that the weak van der Waals forces that stack the planes slightly changed (this has also

4 been reported in the other reports) $[16,22,23]$. Furthermore, the peak positions attributed to the

5 Gp edge planes (e.g., hexagonal (101), (102), (103), (104) and (105)) preferentially broadened

6 and then disappeared with increasing milling time. It indicates the decrease in the crystalline

7 sizes as well as the effective increase in the reactive fine edge surfaces $[17,21]$. Therefore, these

8 changes in diffraction width would be the result of the structural disordering [24]. The dangling

9 bondings at the edges would also be effectively generated along with preserving the layered

10 structures by the milling.

11 Fig. 3 show the Raman spectra of the Gp microparticles without milling and milled for $0.5 \mathrm{~h}, 1$

$12 \mathrm{~h}, 2 \mathrm{~h}$ and $8 \mathrm{~h}$. The changes in the structural order parameters of $I_{D} / I_{G}$ and $I_{D}{ }^{\prime} / I_{G}$ with the reaction

13 time are also shown in Fig. 3. The spectra clearly exhibit the $G, D$ and $D^{\prime}$ bands at around 1350

$14 \mathrm{~cm}^{-1}, 1580 \mathrm{~cm}^{-1}$ and $1600 \mathrm{~cm}^{-1}$, respectively. The $G$ band is attributed to the E2 $\mathrm{g}$ mode of the six-

15 membered ring structures [26], and the band intensity corresponds to the expansion between the

$16 \mathrm{C}-\mathrm{C}$ bonding in-plane direction of the (002) plane. The $D$ and $D$ ' bands, which are thought to be

17 due to the structural disorder, are attributed to the strained lattice disordering by surface

18 modification and substituted impurity atoms along the planes, respectively [27-29]. Thus, the $G$

19 band intensity decreased in this study (Fig. 3 (a)), indicating the breaking of the six-membered

20 ring structures. In contrast, the $D$ and $D^{\prime}$ band intensities increased, suggesting lattice disordering

21 caused by the atomic reactions during the milling. Furthermore, both the $I_{D} / I_{\mathrm{G}}$ and $I_{D}{ }^{\prime} / I_{G}$ values

22 increased with increasing milling time (Fig. 3 (b)). Therefore, the increase in the $I_{D} / I_{G}$ and $I_{D}{ }^{\prime} / I_{G}$

23 values indicates the formation of defective surfaces in the six-membered ring structures that 
1 increase the fine fragmented edge parts. These results correspond to the SEM images and XRD

2 patterns. It was found that the nanoparticles intriguingly consisting of the layered nanostructures

3 as well as reactive fine edge surfaces can be obtained by changing the milling time. According to

4 the previous report [31], the mechanochemical reactions of the Gp with iron oxides have been

5 reported to result in the reduction of the $\mathrm{Gp}(002)$ peak and the production of $\mathrm{CO}_{2}$. In this study,

6 the production of $\mathrm{CO}_{2}$ was not detected by the gas chromatography analysis, only a small amount

7 of hydrogen was produced. This new finding suggests that the $\mathrm{C}-\mathrm{H}$ end groups of Gp effectively

8 reacted each other, thereby causing dissociation of $\mathrm{C}-\mathrm{H}$. This reaction mechanism is now under

9 investigation. The surface reactivity of the Gp nanoparticles with rich dangling bondings and the

10 reaction mechanism will be reported by our group soon.

\section{4. Conclusions}

13 The mechanochemical solid-state control of the nanostructures of plate-like Gp microparticles

14 was achieved in an argon atmosphere. The simple milling process led to the formation of Gp

15 nanoparticles with an aggregated state. The nanostructural analyses by FE-SEM, TEM and XRD

16 suggested that the Gp nanoparticles agglomerated each other to from the aggregation through the

17 dangling bondings on the fine edge surfaces newly formed by the milling. Furthermore, the

18 Raman spectra demonstrated that the ratio of the fine edges to layered inner planes in the Gp

19 significantly increased with the milling. The Gp nanoparticle aggregation, which was

20 successfully prepared by the low environmental burden process without organic solvent, will

21 provide a platform for carbon material functionalization. 


\section{Acknowledgments}

2 This study was partially supported by a Grant-in-Aid for the Adaptable and Seamless 3 Technology Transfer Program through target-driven R\&D (A-STEP: No. AS251Z00303P) of the

4 Japan Science and Technology Agency (JST). The authors gratefully thank Prof. Dr. Yoshio

5 Nosaka for providing the facilities of Nagaoka University of Technology for the Raman 6 spectroscopy. 


\section{References}

2 [1] V.P. David, J.J. Host, M.H. Teng, B.E.J. Hwang, D.L. Johnson, T.O. Mason, J.R. 3 Weertman, Nature 374 (1995) 602.

4 [2] X.L. Dong, Z.D. Zhang, S.R. Jin, B.K. Kim, J. Appl. Phys. 86 (1999) 6701-6705.

5 [3] R. Narayanasamy, V. Anandakrishnan, K.S. Pandey, Mater. Sci. Eng. A 494 (2008) $6 \quad 337-342$.

7 [4] G.B. Jang, M.D. Hur, S.S. Kang, J. Mater. Process Technol. 100 (2000) 110-115.

8 [5] A.C. Ferrari, Solid State Commun. 143 (2007) 47-57.

9 [6] Y. Kobayashi, K. Fukui, T. Enoki, K. Kusakabe, Phys. Rev. B 73 (2006) 10 125415-1-125415-8.

11 [7] R. Saito, M. Yagi, T. Kimura, G. Dresselhaus, M.S. Dresselhaus, J. Phys. Chem. Solids 60 12 (1999) 715-721.

13 [8] M. Nakadaira, R. Saito, T. Kimura, J. Mater. Res. 12 (1996) 1367-1375.

14 [9] V.V. Boldyrev, Russ. Chem. Rev. 75 (2006) 177-189.

15 [10] S. Motozuka, M. Tagaya, T. Ikoma, M. Morinaga, T. Yoshioka, J. Tanaka, J. Phys. Chem. 16 C 117 (2013) 16104-16118.

17 [11] S. Motozuka, M. Tagaya, H. Nishiyama, M. Nishikawa, T. Ikoma, T. Yoshioka, S. Samitsu, J. Tanaka, J. Phys. Chem. C 117 (2013) 9908-9919.

19 [12] M. Beyer, H. Clausen-Schaumann, Chem. Rev. 105 (2005) 2921-2946.

20 [13] M. Tagaya, S. Motozuka, T. Kobayashi, T. Ikoma, J. Tanaka, J. Mater. Chem. 22 (2012) 21 18741-18743

22 [14] L.J. Hardwick, H. Buqa, P. Novak, Solid State Ionics 177 (2006) 2801-2806. 
1 [15] P.K. Chu, L. Li, Mater. Chem. Phys. 96 (2006) 253-277.

2 [16] T.S. Ong, H. Yang, Carbon 38 (2000) 2077-2085.

3 [17] M. Francke, H. Hermann, R. Wenzel, G. Seifert, K. Wetzig, Carbon 43 (2005) 1204-1212.

4 [18] B.K. Yen, B.E. Schwickert, M.F. Toney, Appl. Phys. Lett. 84 (2004) 4702-4704.

5 [19] A.W. Hull, Phys. Rev. 10 (1917) 661-696.

6 [20] Y.X. Zhao, I.L. Spain, Phys. Rev. B 40 (1989) 993-994.

7 [21] P. Scherrer, Nach. Ges. Wiss. Göttingen 26 (1918) 98-100.

8 [22] H. Wakayama, J. Mizuno, Y. Fukushima, K. Nagano, T. Fukunaga, U. Mizutani, Carbon 37 (1999) 947-952.

10 [23] S. Orimo, G. Majer, T. Fukunaga, A. Zuttel, L. Schlappbach, H. Fujii, Appl. Phys. Lett. 75 (1999) 3093-3095.

12 [24] J.Y. Huang, Acta. Mater. 47 (1999) 1801-1808.

13 [25] C.R. Houska, Warren B. E. J. Appl. Phys. 25 (1954) 1503-1509.

14 [26] G. Zhang, S. Sun, Shuhui, D. Yang, J.P. Dodelet, E. Sacher, Carbon 46 (2008) 196-205.

15 [27] G. Chollon J. Takahashi, Compos. Part A 30 (1999) 507-513.

16 [28] H. Murphy, P.T. Papakonstantinou, T.I.T.J. Okapalugo, J. Vac. Sci. Technol. B 24 (2006) $17 \quad 715-720$.

18 [29] M. Endo, C. Kim, T. Karaki, T. Tamaki, Nishinura, Y.; Matthews, M. J. Phys. Rev. B 58 (1998) 8991-8996.

20 [30] H.C. Schiepp, J.L. Li, M.J. McAllister, H. Sai, M. Herrera-Alonso, D.H. Adamson, R.K. 21 Prud'homme, R. Car, D.A. Saville, I.A. Aksay, J. Phys. Chem. B. 110 (2006) 8535-8539.

22 [31] K. Tokumitsu, Solid State Ionics 101-103 (1997) 25-31. 


\section{Figure captions}

2 Fig. 1 Representative (a, b, d, e, g, h) FE-SEM and (c, f, i) TEM images of the GP 3 microparticles $(\mathrm{a}-\mathrm{c})$ without milling and milled for $(\mathrm{d}-\mathrm{f}) 1 \mathrm{~h}$ and $(\mathrm{g}-\mathrm{i}) 8 \mathrm{~h}$. The magnifications 4 are (a) $\times 8 \mathrm{k},($ b) $\times 50 \mathrm{k},(\mathrm{c}) \times 50 \mathrm{k},(\mathrm{d}) \times 30 \mathrm{k},(\mathrm{e}) \times 120 \mathrm{k},(\mathrm{f}) \times 50 \mathrm{k},(\mathrm{g}) \times 60 \mathrm{k},(\mathrm{h}) \times 200 \mathrm{k}$ and (i) $5 \times 250 \mathrm{k})$, respectively.

6

7 Fig. 2 XRD patterns of the GP particles without milling and milled for $0.5 \mathrm{~h}, 1 \mathrm{~h}, 2 \mathrm{~h}$ and $8 \mathrm{~h}$. 8 (b) is magnified by 100 times from (a).

10 Fig. 3 (a) Raman spectra of the GP particles without milling and milled for $0 \mathrm{~h}, 0.5 \mathrm{~h}, 1 \mathrm{~h}, 2 \mathrm{~h}$

11 and $8 \mathrm{~h}$, and (b) the changes in the structural order parameters of $I_{D} / I_{G}$ and $I_{D^{\prime}} / I_{G}$ during the 12 reaction time. 


\section{Fig. 1}
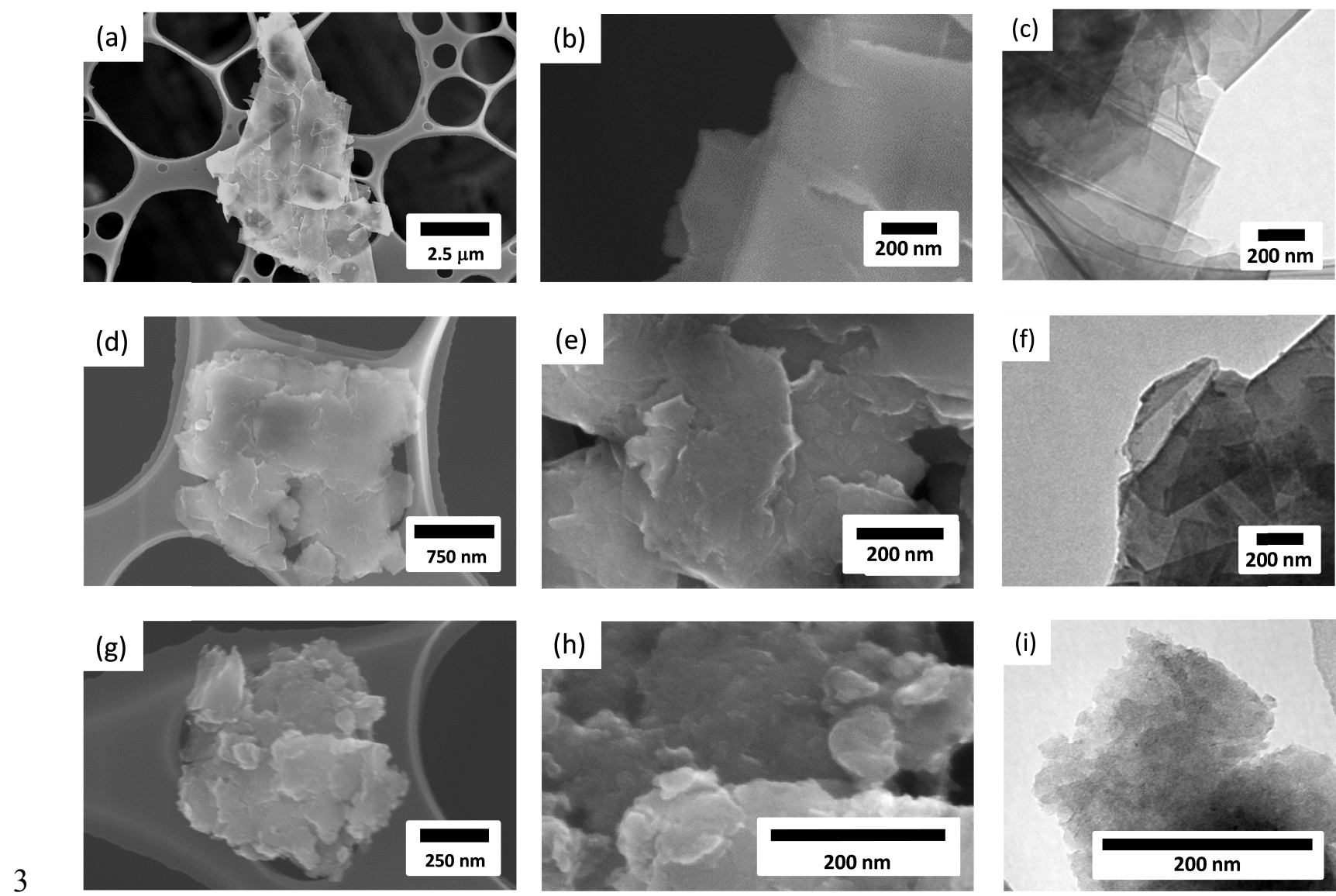
Fig. 2

(a)

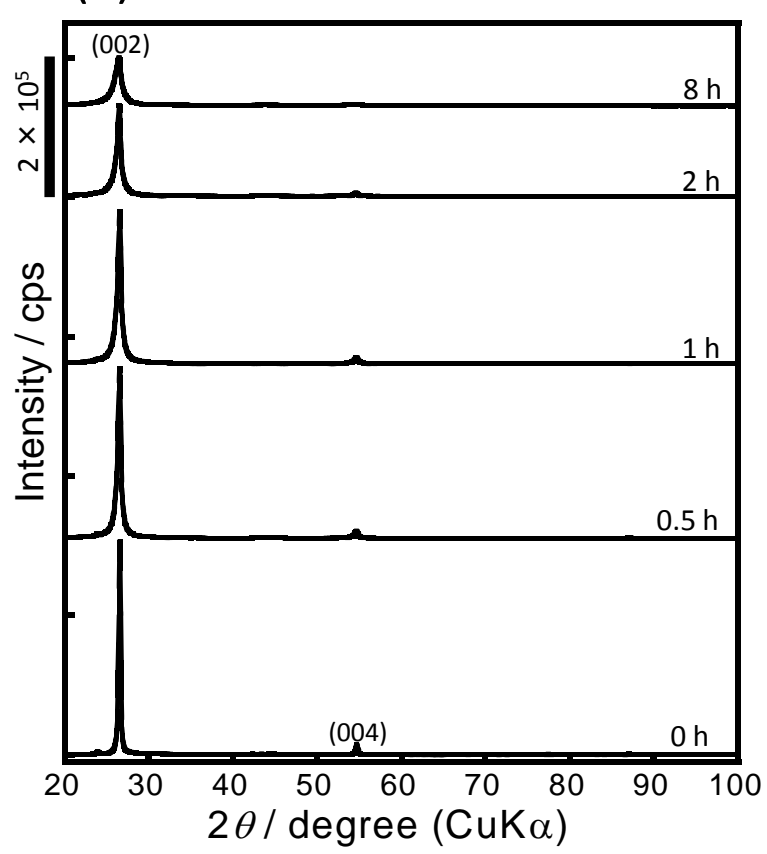

(b)

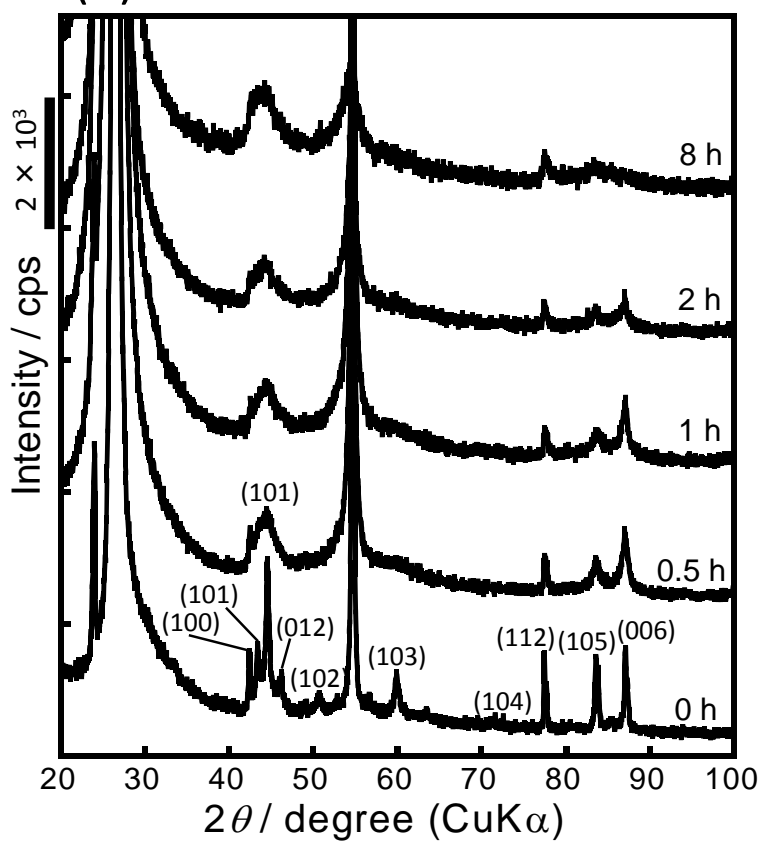


Fig. 3
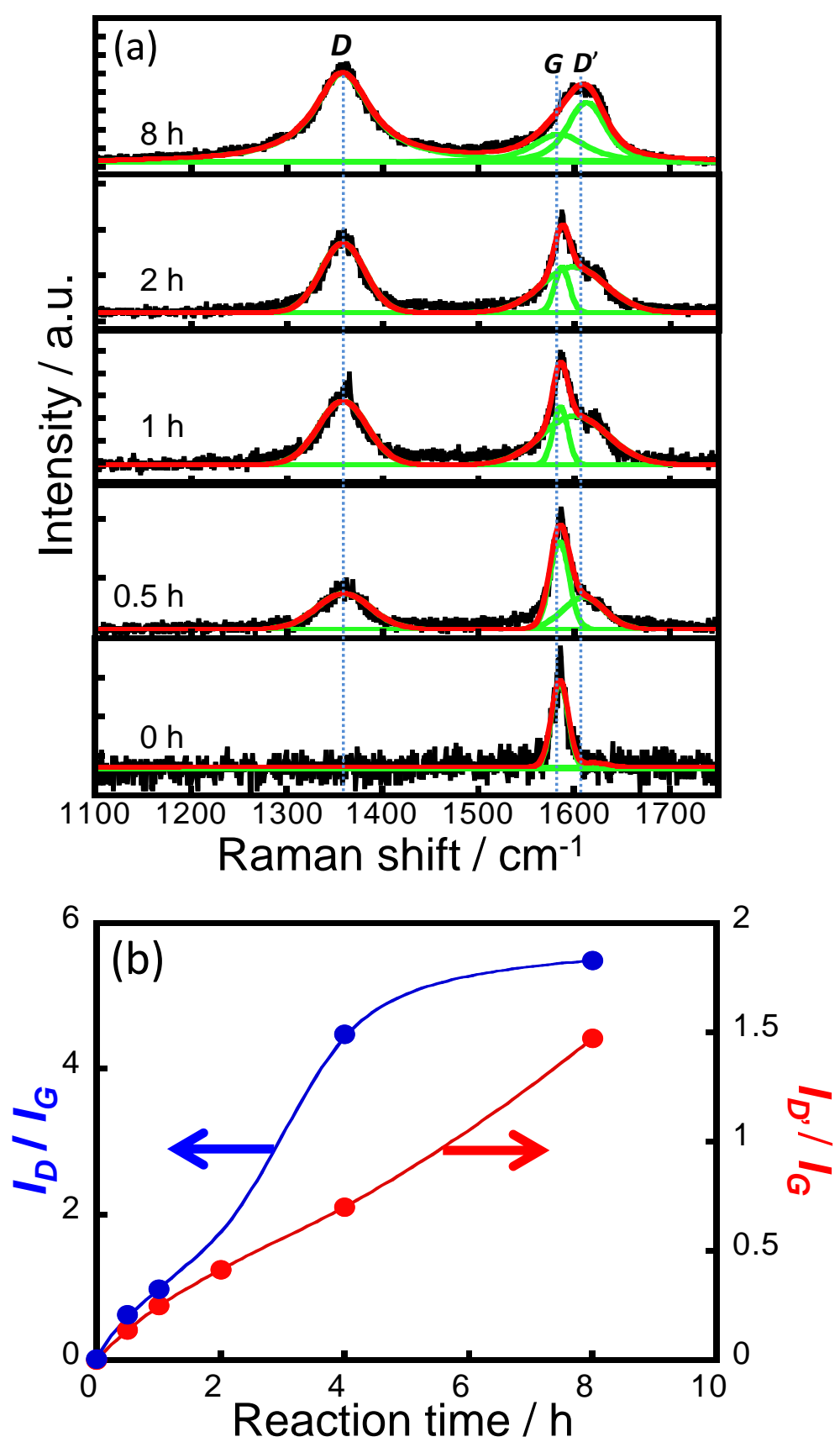


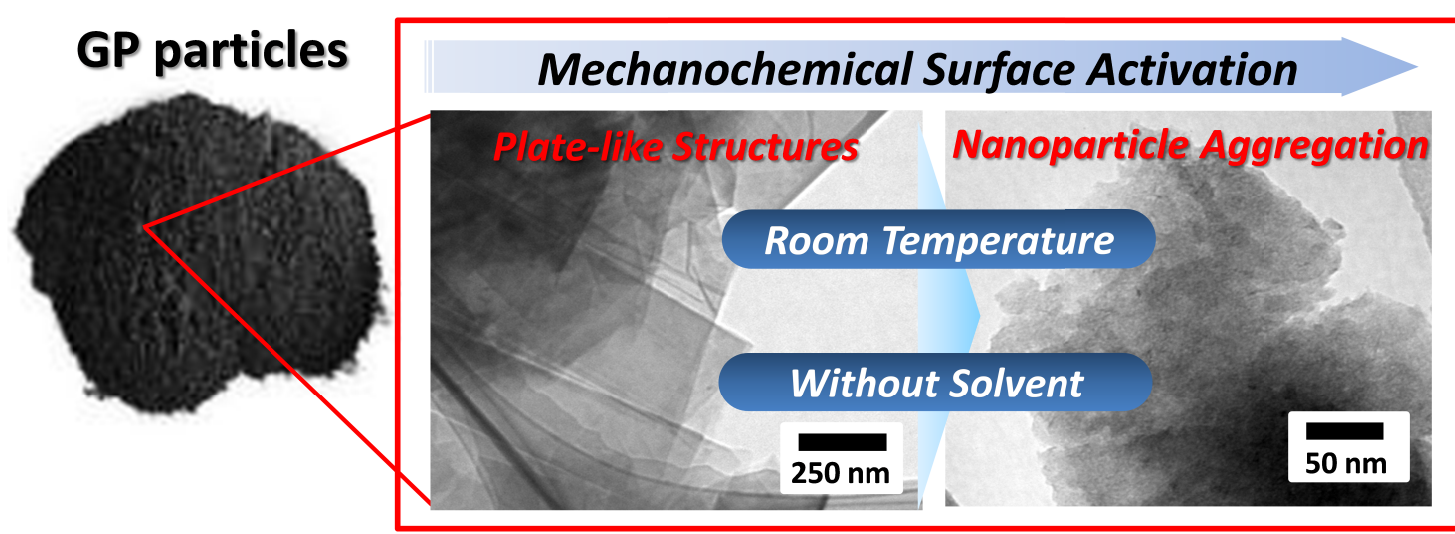

New low environmental burden process 